\title{
Straight alley acquisition drive and ad lib test performance
}

WILLIAM TIMBERLAKE

UNIVERSITY OF MICHIGAN

Five groups of 10 rats received 20 one-per-day acquisition trials in a straight alley. Beginning with Trial 1 either $0,2,4$, 8 , or 20 successive trials were given under high hunger drive and the remainder under low drive. Following eight days of ad lib feeding all Ss received six further test trials. Three of the four groups receiving high drive acquisition trials ran significantly faster in ad lib testing than the group receiving only low drive acquisition trials.

Recent experiments in which straight alley acquisition drive was varied and rat Ss tested under equated drive levels in extinction (Theios, 1963), or in further rewarded trials (Zaretsky, 1966), showed that high acquisition drive produces better test performance than low acquisition drive. The present experiment investigated one possible parameter of this effect by varying the number of high drive acquisition trials Ss received while keeping the total number of acquisition trials constant. It was expected that performance on rewarded ad lib test trials would be a direct function of the proportion of acquisition trials $S$ received under high drive.

\section{Method}

Sixty male hooded rats, 90 days old, were placed on a 22-3/4 $h$ food deprivation schedule 21 days before training. During this period Ss were handled, adapted to the apparatus, and allowed to eat three $.045 \mathrm{gm}$ Noyes pellets in the goal section on the last three days.

The apparatus was a mid-gray wooden straight alley. 6 in. high, 4 in. wide, and $11 \mathrm{ft}$ long. The start section was separated from the alley by two baffles attached such that $S$ had to go under the first and over the second to exit. Start times were measured from the closing of the start box access door to the breaking of a red-filtered photobeam $3-1 / 2$ in. outside the start section. Running times were measured between two photobeams bounding a $5 \mathrm{ft}$ section of runway ending 14 in. from the goal section. On each trial a maximum of $3 \mathrm{~min}$ was allowed for a start time and $6 \mathrm{~min}$ for a running time.

Ss were ranked on the basis of running times obtained the last day of pretraining and distributed randomly within each subset of six to one of six groups. Groups 1 through 5 ran a predetermined total of 20 one-per-day acquisition trials, receiving respectively $0,2,4,8$, and 20 of these trials under high drive and the remainder under low drive. High and low drive were defined as $2 \mathrm{~h}$ before and $2 \mathrm{~h}$ after the regular dally feeding time. Group 6 was placed on ad lib feeding and used to estimate the ad lib body weight of the experimental animals, which under high drive averaged $76 \%$ and under low drive $85 \%$ of ad lib. Following acquisition, Groups 1-5 were also placed on ad lib feeding for eight days until no group differed significantly in average weight from Group 6. Ss then received six further runway trials, one trial per day, under the ad lib feeding conditions. These test trials were given at a different time of day than the acquisition trials in order to minimize any effect of hunger responses conditioned to time dependent environmental cues (Birch et al, 1958). All trials were rewarded with three $.045 \mathrm{gm}$ Noyes pellets.

\section{Results and Discussion}

Mean log running time, the primary dependent measure, is shown over blocks of two trials in Fig. 1 as $\log 100-$ mean $\log x$. The shifts in acquisition drive (marked in Fig. 1) were not uniform in their effect on the performance of the shift group. Shift 1 (Group 2) showed an average decrease in running time of $.26 \mathrm{sec}$, Shift 2 (Group 3) an average decrease of $.36 \mathrm{sec}$, and Shift 3 (Group 4) an average increase of .06 sec. Only the change associated with Group 3 was significant when evaluated against the hypothesis of no change $(t=2.44$, df $=9, p<.05)$. However, a different picture emerged when the performance changes were evaluated relative to the concurrent performance change for Group 5, the group continuing under the original high drive conditions. Group 2 showed an average relative decrease in running time of $.07 \mathrm{sec}$, Group 3 an average relative decrease of .31 sec, and Group 4 an average relative decrease of $.28 \mathrm{sec}$. No shift group performance change differed significantly from

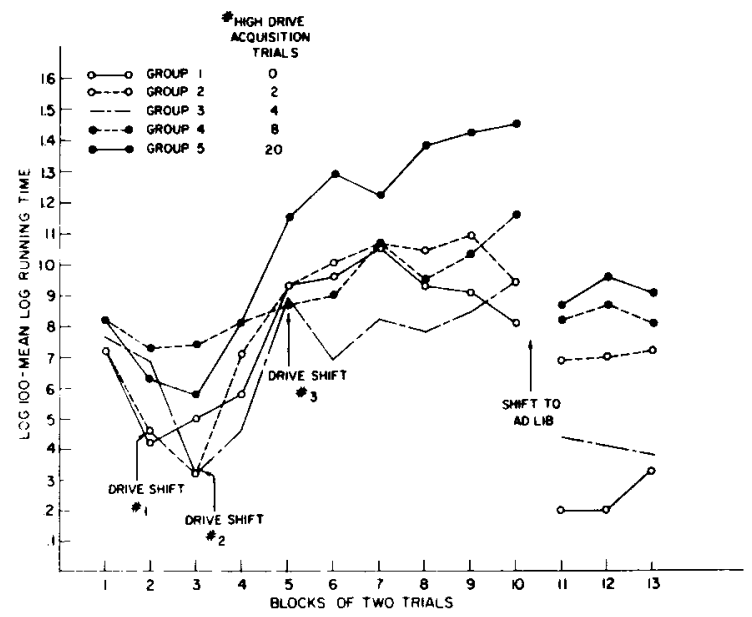

Fig. 1. Mean log nunning time in acquisition and ad lib testing for Groups 1-5. 
that of Group 5, though the t's for Shifts 2 and 3 reached the .10 level. When these last two shifts were evaluated by trials rather than trial blocks the effect of Shift 3 was significant at the .01 level ( $t$ $=2.83, \mathrm{df}=18$ ). Hence, the size of the absolute performance decrement associated with a down shift in drive perhaps decreases as the number of pre-shift trials increases; on the other hand, the size of the relative performance decrement associated with a down shift in drive, appears to increase as a function of the number of pre-shift acquisition trials.

Examination of the shift to the ad lib condition in Fig. 1 indicates that in addition to the number of pre-shift trials, a second variable, number of previous drive levels experienced, may also affect the size of the performance change associated with a down shift in drive. Difference scores obtained by subtracting an S's score on each block of ad lib trials from the average of its scores on the last three acquisition blocks indicated that the groups receiving two levels of acquisition drive showed less decrement from acquisition to ad lib testing than the groups experiencing only one acquisition drive level. Since a simple analysis of variance showed no trials effect ( $F<1$, df $=2 / 98$ ), these scores were summed over trials yielding: Running times (in logs) of groups recelving one acquisition drive level-Group $1=19.27$, Group $5=14.92$; running times of groups receiving two drive levels-Group $2=9.46$, Group $3=13.37$, Group $4=6.47$. A planned comparison of Groups 1 and 5 with Groups 2, 3, and 4 yielded an F significant at the .01 level $(F=14.64, d f=1 / 45)$. In addition, when the performance change of each group was evaluated separately against the hypothesis of no change, all were significant at the .01 level.

The effect of varying the number of high drive acquisition trials can be seen most clearly in the ad lib test blocks. All groups receiving high drive acquisition trials, regardless of number, ran faster than the low acquisition drive group. Further, excepting one reversal between Groups 2 and 3, average running times increased as the number of high drive acquisition trials Ss received increased. An analysis of variance on the three ad lib trial blocks failed to yield a significant groups effect ( $F=2.46$, $d f=4 / 45$ ). However, a Dunnett's test of all means vs a control (Group 1, the low acquisition drive group), showed that Groups 2, 4, and 5 differed significantly from Group 1 at the .01 level $(t=3.05,3.90,4.42$, df =45), but Group 3 did not $(t<1, d f=45)$. There was no trials effect. Some differences between high and low drive acquisition groups can also be seen in the last three or four acquisition trial blocks where all groups receiving high drive trials except Group
3 are faster than Group 1. An analysis of variance over the last three acquisition blocks showed a significant groups effect $(F=3.49, \mathrm{df}=4 / 45, \mathrm{p}<.05)$; but a Newman-Kuels test of all possible differences between single means indicated only that Group 5 (the high acquisition drive group) ran faster than all other groups which did not differ significantly among themselves. There was again no trials effect.

The start times did not show the same results as the running times, perhaps due in part to the unique nature of the start section. At the end of training the only difference indicated is that Ss experiencing two acquisition drive levels started significantly more slowly than Ss receiving only one acquisition drive level. In ad lib testing there was no consistent groups effect. However, similar to the case for running times, calculation of difference scores between the end of acquisition and ad lib testing indicated that Ss experiencing two levels of acquisition drive showed a smaller absolute performance decrement when shifted to ad lib than did the single acquisition drive groups. A planned comparison of the performance of Groups 1 and 5 with Groups 2, 3, and 4 resulted in $F=$ $6.06(\mathrm{df}=1 / 45, \mathrm{p}<.05)$.

In brief, running times in ad lib test blocks and to a lesser extent in the last few acquisition blocks showed that high acquisition drive produces better performance than low acquisition drive when testing occurs under a common ad lib drive level. Some support was obtained for the hypothesis that performance under ad lib testing should be proportional to the number of high drive acquisition trials Ss received; however, this effect was confounded with or perhaps partially mediated by the smaller performance decrement from acquisition to ad lib testing for Ss experiencing two levels of acquisition drive (an effect shown also in the start times). This smaller decrement, when coupled with the relative positions of the groups at the end of acquisition, could account for the running times of Groups 2-4 ordering themselves between those for Groups 1 and 5 in ad lib testing.

\section{References}

BIRCH, D., BURNSTEIN, E., \& CLARK, R. A. Response strength as a function of hours of food deprivation under a controlled maintenance schedule. J. comp. physiol. Psychol., 1958, 51, 350-354.

THEIOS, J. Drive stimulus generalization increments. J. comp. physiol. Psychol., 1963, 56, 691-695.

ZARETSKY, H. H. Learning and performance in the runway as a function of the shift in drive and incentive. J. comp. physiol. Psychol., $1966,62,218-221$.

Note

1. This research was conducted while the author held an NSF predoctoral fellowship. It was partly supported by NSF Grant GB 2910 to Dr. David Birch, whom the writer wishes to thank for his critical assistance. 